\title{
Using Action Research for Complex Research Initiatives
}

\author{
Mardé Greeff and Louis Coetzee \\ Meraka Institute of the CSIR \\ P.O. Box 395 \\ 0001 Pretoria \\ South Africa \\ Email: \{mgreeff, lcoetze1\}@csir.co.za
}

\begin{abstract}
Training methods and the transfer of knowledge and information has essentially remained unchanged for many years. With the pervasiveness of information and communication technology (ICT), an opportunity has been created to fundamentally change the status quo of training. This paper presents one approach of using ICT through the innovative combination of factors such as a person's abilities, styles and preferences with a multimodal interface to facilitate enhanced training. However, many ways exist to guide the research process of such a complex research initiative. Action research is one research method that lends itself to these complex projects. The paper uses the Ability Based Technology Interventions (AbTi) research project as a case study to analyse the effectiveness of the action research methodology. The paper describes the accumulated learning as obtained from the AbTi research project, as well as the action research methodology used, in order to ultimately be in a position to effect a fundamental change in training. It is found that action research can be an effective methodology for research initiatives of a complex nature.
\end{abstract}

Keywords: multimodal, ability based training system, action research, learning style, sensory and perceptual preference

\section{INTRODUCTION}

In a developing world context the transfer of skills and associated training is of critical importance. Persons with disabilities not only experience common training challenges, but also face many additional needs and barriers that they have to overcome. Integration of Information and Communication Technologies (ICT) in the daily lives of many developing world citizens are opening up opportunities to improve the training experience of a substantial number of people.

The ultimate goal of the Ability based Technology Interventions (AbTi) project is to develop a fully functional ability based training platform that facilitates training through technology interventions utilizing different modalities, that are based on the abilities, learning style, sensory and perceptual preferences and literacy level of a trainee.

The project's research methodology is based on the action research method. Action research aims to address the concerns of people in a problematic situation through collaboration within a mutually agreed upon ethical framework [1]. Action research enables stakeholders and members of the disabled community to participate in the project and to provide valuable input [2]. Action research promotes participation of all parties involved, and embodies an approach that incorporates the formulation of a theory, as well as interventions and actions that promote change [3], [4]. Therefore, the area of Information Systems Development can successfully apply action research to enrich the standard methods of software engineering.

Our contributions in this paper are the following:

- Illustrating, by using the AbTi project as a case study, how action research can be used for complex research initiatives;

- Providing one method that can be used to develop a multimodal training platform; and

- Describing the research areas that should be investigated for such a multimodal training platform.

The rest of the paper's layout is as follows: Section II discusses input and output modalities and provides information on related work, highlighting gaps in the current literature. The action research method is discussed in Section III. Section IV presents a case study to demonstrate how action research can be used in a complex research initiative. Section V comments on observations that were made while using the action research methodology in a complex research initiative. Finally, Section VI presents conclusions with regards to this research.

\section{BACKGROUND}

Section II-A provides information with regards to input and output modalities and Section II-B discusses related work.

\section{A. Input and Output Modalities}

In human-computer interaction, a modality can be described as the sense through which a user can receive output from a computer and the way that a computer can receive input from a user, through a sensor or device.

1) Input Modalities: Input modalities or modes are ways in which a computer can receive input from a user through a sensor or device. Figure 1 illustrates various input modalities.

Some of these input modes' data has to be converted to characters/text or pointer events by a specialised application, for example a speech recogniser or gesture recogniser; while other input modes automatically output characters/text or pointer events. These events or characters are used as input into the Integration Manager that integrates and manages data from 


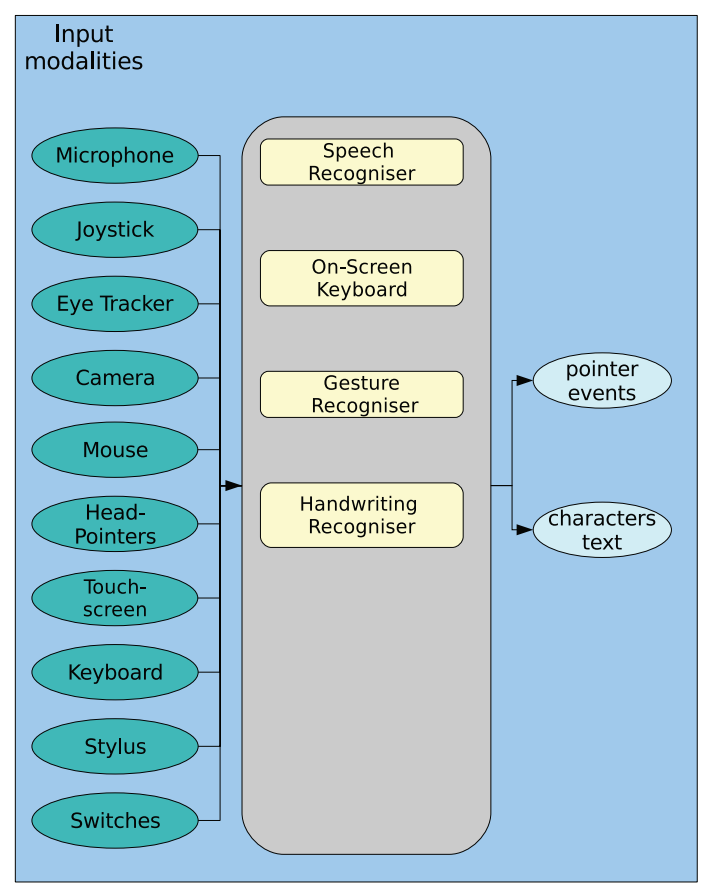

Fig. 1. Overview of various input modalities

and to the various input and output modalities, as illustrated in Figure 2.

2) Output Modalities: Output modes or modalities are the sense through which a user can receive output from a computer. The most frequently used output modalities are visual, auditory and somatic (tactile and haptics). Various output modalities are illustrated in Figure 3.

\section{B. Related Work}

Each person has unique skills and abilities. Accordingly each person's preferences when interacting with a system will vary. One mode of input will therefore not be sufficient to address the diverse needs of various users, especially users with disabilities. When a system is designed for users with various disabilities, as well as different degrees of disabilities, it becomes apparent that more than one modality should be used for input into and interaction with the system, as well as output from the system.

Many sensory modalities exist, of which some are auditory with voice and non-speech input, visual with gaze and image capture as input, and haptic or kinestetic with touch and hand, head or body movements as input [5]. Gestural interfaces have been used to enable persons with motion impairments to interact with a system as easy and efficient as possible [6], [7]. Multi-modal input for people that use Augmentative and Alternative Communication (AAC) devices includes a combination of head pointing and speech input [8]. Visual representations of data, for example graphs, are often used when analysing data or during presentations. However, people with visual impairments cannot access these visual representations or get the same information from the visual representations as sighted people. One way of communicating this information to these users is through audio, but speech output limits the navigation and access of the visual data. To address these limitations, additional feedback can be provided to the visually impaired users through haptic or tactile displays [9], [10], [11].

A flexible multimodal interface enables the user to choose a combination of modalities or to switch to a better suited modality, depending on their preferences and abilities [12], [13]. Aloise et al. investigated the implementation of a system using various input modalities, such as a mouse, joystick, voice recognition, eye tracker and Brain-Computer-Interfaces (BCIs), for people with neuro-motor impairments to improve or recover their mobility [14]. Parés et al. discuss an adaptive physical environment that enables children with severe autism to interact with the environment through multimodal stimuli.

The learning content or material can be presented to the learner through various modalities. At the same time, the learner can interact with the material through different modalities. Therefore, in order to select the most appropriate modalities for a user, his/her learning style should be identified. The Dunn and Dunn Learning-style model specifies 21 elements that influence how people learn [15]. These elements are divided into five stimuli: environmental, emotional, sociological, psychological and physiological stimulus. The environmental stimulus takes into account the environmental preferences of the learner, for example whether the learner prefers to learn in bright or dim light, with or without sound or in a warm or cool room. The emotional preferences of the learner indicates whether s/he requires structure, and whether the learner is motivated, responsible and persistent. The sociological elements highlight whether a learner prefers to learn by him-/herself, in a pair, with peers, in a team, with an authoritive figure present or a combination of these. Physiological preferences takes into account whether a learner prefers to receive learning material in an auditory, visual, tactual or kinesthetic way. Psychological stimulus describes whether a person needs to first understand the concepts and then concentrate on the details (global learner) or prefers to learn the material in a sequential manner that gradually builds towards his/her conceptual understanding.

VanEtten highlights that the human can receive information through six modalities [16] and regards auditory and visual modalities as the most important for the academic learning for people with learning disabilities. According to VanEtten the other modalities will be used mostly to support the learning.

ICT can assist in the empowerment of people with disabilities by enabling them to acquire new skills or to improve their existing skills [17]. Research has been done with regards to how multimodal systems can be used for training, especially training persons with disabilities. Baldi is a computer-animated tutor that teaches grammar and vocabulary to children with autism through both visual and audio modalities [18]. 


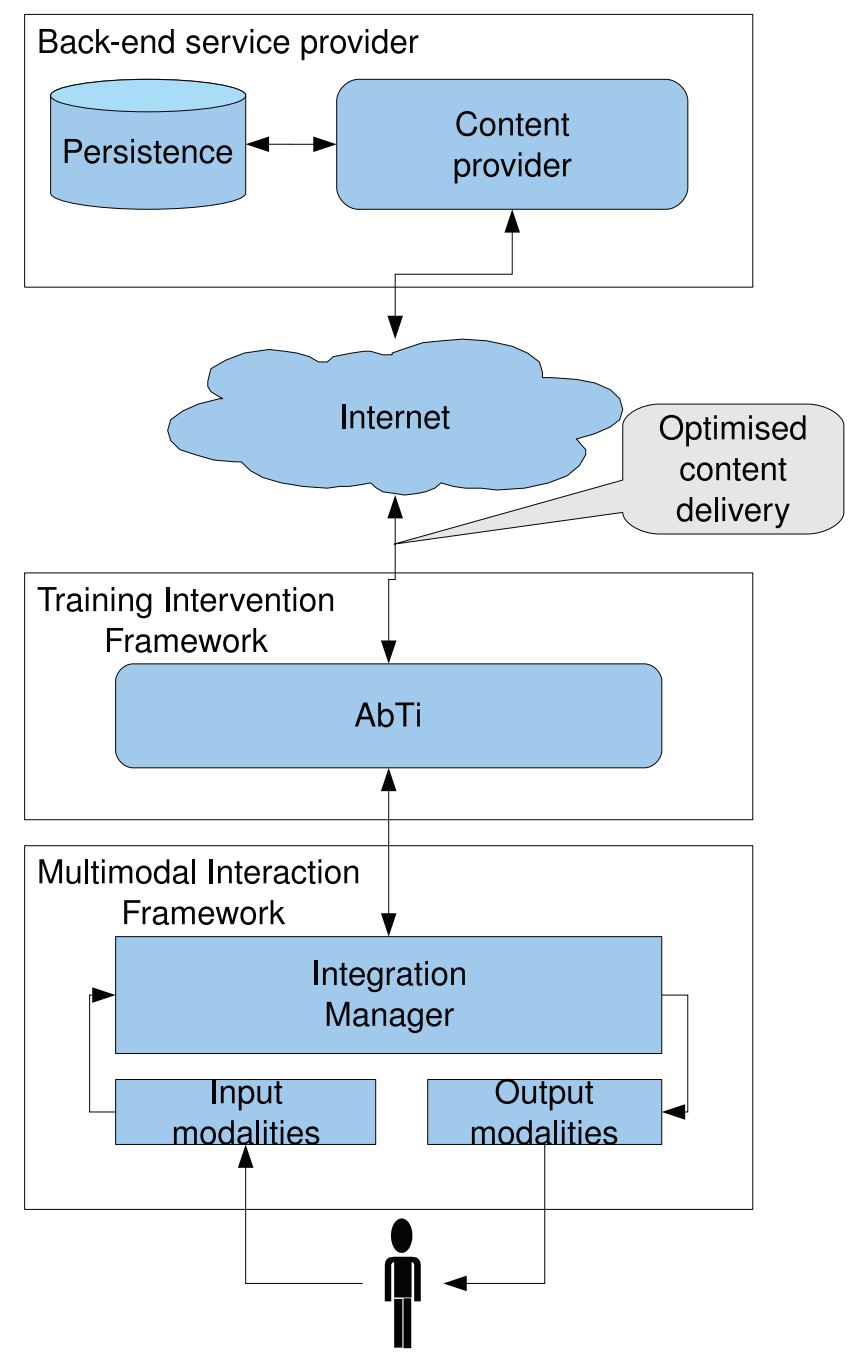

Fig. 2. Overview of the AbTi system

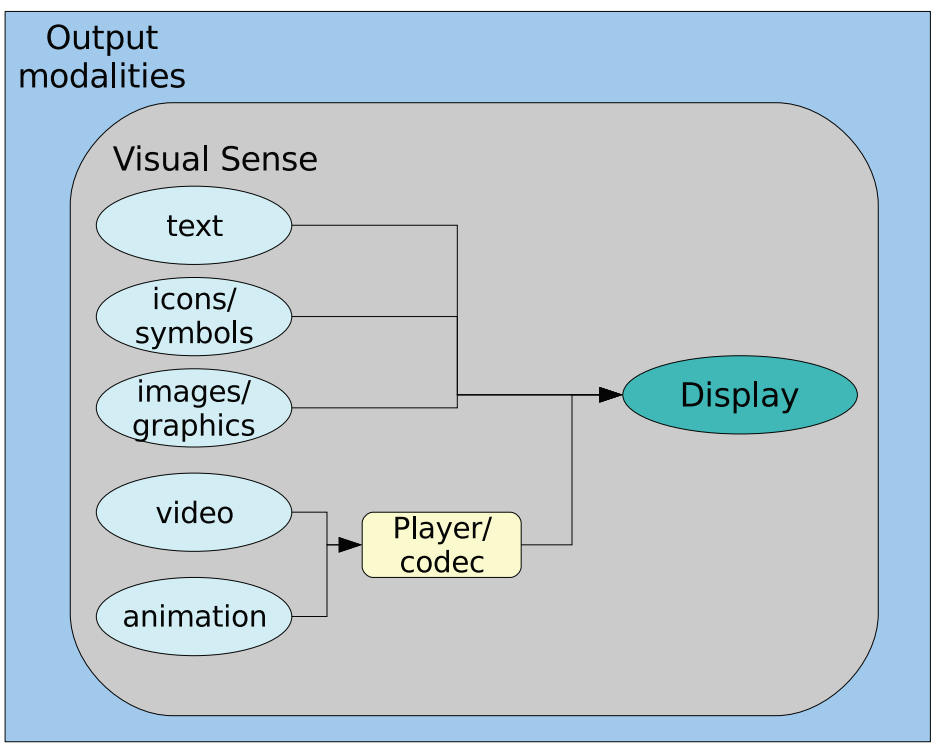

Fig. 3. Overview of various output modalities 
TEACH-M is an instructional package that facilitates learning and retention of persons with severe memory impairments [19]. Douglas provides an overview of the usage of ICT for the education of children with visual impairments [20]. Research has also been done with regards to how people with traumatic brain injury can learn using the Internet [21]. Kabassi and Virvou describes a system that assists adults to learn, by observing the user and when it suspects that the user is experiencing problems, it adapts the tutoring [22].

More recently, Doulgeraki et al. presented a new methodology for adaptable web-based systems. Their methodology's user information component takes into account the disability, language and web familiarity (or experience) of the user [23].

In the last few years action research is being used more frequently in the ICT domain [24]. Chetty et al. discussed a voice over IP (VoIP) application with culturally sensitive and locally relevant content for a specific rural community that was developed using critical action research [25]. Hearn and Foth discussed the action research for ICT and multi-media projects [24]. However, most of the work published are about the development of a specific ICT system or product and not about complex research initiatives that may stretch over 3-5 years.

From the above the following gaps in the literature can be identified:

- Much research has been done with regards to using multimodal input or output for a specific disability. However, using a multimodal system for users with various disabilities and varying degrees of the disabilities, has not been researched extensively and more research is required in a variety of areas, such as: which modalities are best suited for which abilities and skills of the user; which modalities are best suited for the various learning styles that the users can have, etc.

- Most of the multimodal systems are configured for persons with a specific disability, e.g. motion impairments or learning disabilities. However, when you want to research how to adapt training content according to the user's abilities, learning style and literacy level, much more research should be done into the best ways of presenting the content in the most appropriate way.

- Recent work takes into account the disability, language and web experience of users when creating the user profile for an adaptable web-based system. However, the authors don't take into account the learning style and sensory and perceptual preference of the user.

- Many research methods exist that can be used to guide the research process in complex research initiatives. However, not many experiences (case studies) or "lessons learned" are published with regards to using the various kinds of research methods for complex initiatives.

Section IV discusses how the AbTi project addresses these identified gaps. The next section provides a short overview of the action research method, which is explained in more detail through a case study in Section IV.

\section{ACTION RESEARCH}

The action research method aims to contribute to basic and applied research in a specific domain, while also addressing a practical concern of people in a specific situation [26]. Action research enables effective collaboration between practitioners and researchers and consists of a cycle of distinct phases. The phases of action research are:

1) Diagnosing phase where the problem is identified and defined.

2) Action planning phase where alternative solutions and actions are considered based on theory.

3) Action taking phase where a course of action or intervention is selected and implemented. This is where prototypes, technology and technology demonstrators are developed.

4) Evaluating phase where the consequences and impact of the action or intervention are studied or observed.

5) Specifying learning phase where research findings and evaluation results are documented and published. The learning is fed into a new cycle that restarts at a new diagnosing phase.

In complex research initiatives, each phase will consist of a number of activities. Most research initiatives will require more than one action research cycle. The next section presents a case study, discussing how the action research method has been applied to the AbTi research project.

\section{Case Study: Using Action Research For A COMPLEX RESEARCH INITIATIVE}

This section describes how action research has been applied to the AbTi project. Section IV-A provides an overview of the AbTi project and highlights research areas that should be investigated when developing an ability based multimodal training system. In addition, Section IV-B highlights how the action research methodology has been applied to the AbTi project.

\section{A. AbTi project}

The goal of the AbTi research project is to investigate how the acquisition of new skills and knowledge can be facilitated and improved through the use of multiple modalities. The selection of modalities is based on the unique abilities, learning style and literacy level of a trainee. The users of the system will be persons using technology to acquire new skills, and training providers that use technology to teach skills. The training material must be presented in such a way that a person with limited abilities (for example a person with disabilities) can access and interact with the training material utilizing his/her abilities optimally through the appropriate modalities. Therefore, the training content is presented to a trainee in modalities that are appropriate for his/her physical abilities, literacy level and learning style. 
1) Research Areas required for Multi-modal Training: In order to provide ability-based training, the following areas should be investigated:

- How people learn;

- Determining the profile of a trainee;

- Determining which modalities should be utilised to enable ability based training;

- Determining the optimal mapping between trainee profiles and presentation modalities; and

- Determining the format in which the training content should be stored and transformed to support multimodal presentation.

Each of these research areas is discussed in more detail below.

\section{How people learn}

Training needs must be determined for various abilities, learning styles, and literacy levels, i.e. various trainee profiles. The different abilities of persons with various disabilities, such as hearing-, visual-, speech-, mobility- and intellectual impairments, must be addressed.

All people have natural perceptual learning preferences: $\mathrm{read} / \mathrm{write}$ learners learn most effectively by reading information; visual learners prefer information illustrated through graphs; auditory learners learn more effectively by listening to instructions or information; and kinesthetic learners learn best through experience and demonstrations. Futhermore, people also have natural learning styles: an activist learns most effectively when $\mathrm{s} / \mathrm{he}$ is in the middle of a new experience or can help to solve a new problem; reflectors prefers to observe others and have time to review what they have learned at the end of the day; theorists learn more effectively when they have the opportunity to question concepts and ideas; and pracmatists learn best when they are provided with techniques that saves them time and they are able to try out new techniques and provide feedback on these new techniques. Therefore, the best way of presenting the training material for trainees with each of these different perceptual learning preferences and learning styles must be determined.

The literacy level of a prospective trainee will also impact the effectiveness of the training. Therefore, the best way of presenting training material in simplified language to accommodate the literacy level of a trainee must be determined. This research will also benefit persons with hearing (many Deaf people in developing countries cannot read or write since Sign Language is their first language) or intellectual disabilities.

\section{Determining the profile of a trainee}

In order to present training in formats and modalities appropriate for the trainee's abilities, learning style, and literacy level, the profile of each trainee must be determined. Various methods and models of assessing the abilities and literacy levels of persons are researched, such as: evaluation methods, assessment methods and the specification of options for preferences. Methods to assess the effective learning style of a trainee must also be determined. Internationalisation and localisation should also be investigated to enable trainees that are not proficient in English, to receive training in his/her first language. This will also benefit users in the broader African and International community.

\section{Determining which modalities should be utilised to enable} ability based training

Possible modalities that can be used for input into, interaction with and output of training material must be determined. Refer to Section II-A for more information.

\section{Determining the optimal mapping between trainee profiles and presentation modalities}

The mapping between trainee's profile (his/her abilities, learning style, and literacy level) must be determined. Therefore, prototypes of different modalities will be developed and evaluated to determine the usability and effectiveness of the modality for a specific profile.

Determining the format in which the training content should be stored and transformed to support multimodal presentation

The format for effective storing, retrieving and presentation of training content to support different modalities must be determined. Existing standards and how the standards can be adjusted to include the requirements for different modalities will be investigated. Through the standardisation of content formats, independent training providers will be empowered to create content material containing the various modality presentation formats. Investigation is also required into how content can be transformed into different modalities to match the profile of a trainee. The models to simplify the language of the training material to match the literacy level of the trainee must also be developed.

2) Technology required for Multi-modal Training: Figure 2 presents an overview of the functional elements required for the creation of a complete multimodal training system.

The back-end service provider provides the storage and dissemination of content, a typical implementation would be a web application server.

The training intervention framework consists of a variety of components, the three most important being:

- A mechanism to retrieve content and transform it into the appropriate combination of modalities for the specific trainee profile;

- A mechanism to dynamically adapt the user interface to render the various content formats; and

- A mapping module that determines the most appropriate combination of input- and output modalities based on the trainee's profile. This can be implemented through the utilization of a Bayesian network.

The multimodal interaction framework allows for the synchronized rendering of content in the chosen output formats, whilst it also allows for the fusion of different inputs obtained from the user to control the dynamic flow of the training content. 
TABLE I

ACTIVITIES PER PHASE FOR EACH RESEARCH CYCLE

\begin{tabular}{|c|c|c|}
\hline Phase & Cycle One & Cycle Two \\
\hline Diagnosing & $\begin{array}{l}\text { - Investigate the research areas to be explored } \\
\text { in the project and determine the scope of } \\
\text { subsequent phases and activities. } \\
\text { - Determine training needs per ability, disabil- } \\
\text { ity, learning style and literacy level. } \\
\text { - Determine ethical issues and develop ethics } \\
\text { process to be implemented before any contact } \\
\text { with users are initiated. }\end{array}$ & $\begin{array}{l}\text { - Revisit training needs per ability, learning } \\
\text { style and literacy levels based on learning } \\
\text { gained in the first research cycle. }\end{array}$ \\
\hline Action Planning & $\begin{array}{l}\text { - Investigate existing work, trends, role players } \\
\text { \& establish collaboration mechanisms and } \\
\text { partnerships. } \\
\text { - Investigate language representation \& adjust- } \\
\text { ment needs for different literacy levels. } \\
\text { - Investigate different modalities available for } \\
\text { training purposes. } \\
\text { - Investigate content formats for multiple } \\
\text { modalities. } \\
\text { - Determine methods for assessing abilities, } \\
\text { learning styles and literacy levels. } \\
\text { - Explore technology to wrap and present mul- } \\
\text { timodal content streams. } \\
\text { - Investigate models and methods for mapping } \\
\text { of modalities to different abilities, learning } \\
\text { styles and literacy levels. } \\
\text { - Plan action taking phase. }\end{array}$ & $\begin{array}{l}\text { - Plan action taking phase based on updated } \\
\text { needs and learning gained during the first } \\
\text { research cycle. } \\
\text { - Conduct further investigation as identified } \\
\text { during the first research cycle. }\end{array}$ \\
\hline Action Taking & $\begin{array}{l}\text { - Develop prototypes to explore and test differ- } \\
\text { ent modalities for input into training material. } \\
\text { - Develop prototypes to explore and test dif- } \\
\text { ferent modalities for interaction with training } \\
\text { material. } \\
\text { - Develop prototypes to explore and test dif- } \\
\text { ferent modalities for presentation of training } \\
\text { material. } \\
\text { - Develop models for assessing abilities, learn- } \\
\text { ing styles and literacy levels. } \\
\text { - Develop prototypes to test the models for } \\
\text { assessing abilities, learning styles and literacy } \\
\text { levels. } \\
\text { - Develop models to map trainee profiles with } \\
\text { appropriate modalities. } \\
\text { - Develop prototypes to test the mapping of } \\
\text { trainee profiles with appropriate modalities. } \\
\text { - Develop models for transforming training } \\
\text { material to support the various modalities. } \\
\text { - Develop prototypes to test the transformation } \\
\text { of training material to support the various } \\
\text { modalities. }\end{array}$ & $\begin{array}{l}\text { - Exploratory development of new technology. } \\
\text { - Refinement of technology developed in the } \\
\text { first cycle based on learning gained and ad- } \\
\text { ditional knowledge acquired. }\end{array}$ \\
\hline Evaluating & $\begin{array}{l}\text { - Develop evaluation strategies and methodolo- } \\
\text { gies. } \\
\text { - Evaluate developed prototypes and technolo- } \\
\text { gies. }\end{array}$ & $\begin{array}{l}\text { - Evaluate developed technology. } \\
\text { - Evaluate the impact of technology in the } \\
\text { application domain. }\end{array}$ \\
\hline Specifying Learning & $\begin{array}{l}\text { - Document and publish findings. } \\
\text { - Compile progress reports. }\end{array}$ & $\begin{array}{l}\text { - Document and publish findings. } \\
\text { - Compile final report. }\end{array}$ \\
\hline
\end{tabular}




\section{B. Using Action Research in the AbTi Project}

The action research method is used in the AbTi project to guide the research process. The AbTi project consists of two action research cycles. The first action research cycle contains all initial activities in all the phases, while the activities in second cycle are defined based on the findings and learning gained in the first cycle. The specific activities that take place during each cycle, are illustrated in Table I. Below, each of these phases are described in more detail.

\section{Diagnosing phase}

In the diagnosing phase of the project, the training needs are determined for persons with different disabilities, abilities, learning styles and literacy levels. This research is conducted in partnership with training specialists, educators, educational kinesiologists, and specialists in the disability domain.

\section{Action planning phase}

The action planning phases of the project comprise of multidisciplinary research activities, including literature reviews; data collection; document analysis; collaboration with national and international experts and technical research. Topics to be investigated include:

- Existing activities and trends in eTraining with special emphasis on the application of different modalities.

- Identification of national and international role players for possible collaboration; Language representation and adjustments needed to accommodate trainees with different literacy levels.

- Investigation into the use of different modalities for input into, interaction with and output of training material.

- Evaluation of feasibility, effectiveness and usability of different modalities for training purposes.

- Investigation into the effective creation, storing, retrieving and presenting of training material for various modalities including existing standards.

- Methods of assessment of abilities, learning styles and literacy level of a prospective trainee.

- Human-computer interaction issues regarding multimodal applications and interfaces.

- Technology to wrap multimodality content streams and present it in an effective, efficient manner.

- Issues regarding the optimised mapping of the abilities, learning style and literacy level of a trainee with the appropriate training process and modalities.

\section{Action taking phase}

The action taking phases of the project consist of technology research and exploratory development of prototypes and technology building blocks, to test the theory and evaluate the feasibility, efficiency and usability of the models and modalities. Technology to be developed include, but is not limited to:

- Prototypes of different modalities for input into, interaction with, and output of training material based on the ability of a trainee.
- Models for assessment and evaluation of abilities and literacy levels of trainee.

- Models and prototypes to map the profile of a trainee to the appropriate modalities.

- Models and prototypes for storing, transforming and presenting training material to support the various modalities.

The activities in this phase are conducted using a user centred approach, which dictates frequent interaction with possible users of the system and other stakeholders.

\section{Evaluating phase}

The evaluating phases of the research consist of evaluation studies with identified trainees, utilising a variety of researched and developed elements. Evaluation includes usability testing in usability laboratories, effectiveness evaluations, evaluation by experts in the domain and technical evaluation of the performance and efficiency of modalities. Evaluation frameworks for different modalities are developed in these phases. Qualitative research methods are used to assess the impact of the research on the efficiency of training of persons with different abilities, learning styles and literacy levels. Research methods include ethnographic studies, data collection, document analysis, interviews and usability evaluation.

\section{Specifying learning phase}

The specifying learning phase of the project give researchers the opportunity to document their findings for publication purposes, as well as using the findings as input into a new cycle of action research.

The next section comments on observations made and "lessons learned" while using the action research methodology in the AbTi project.

\section{Comments on ACtion Research AS A METHODOLOGY}

Various approaches exist to conduct and guide research of complex nature. Action research is used in the AbTi project. It has been found that the various phases allow for the introduction of effective structure, which effectively guides the research effort. One element which has detracted from the effectiveness is that the specifying learning phase (refer to Section III) is at the end of the cycle. Thus, research outputs are only generated after the research effort. This can be problematic, since research initiatives are often evaluated during the course of the project, with the expectations that research outputs are generated earlier. One way to address the problem, is to plan the various cycles of action research according to the expected deadline, to ensure that the specifying learning phase is completed before the evaluation date. However, this may not always be possible to achieve. Complex research initiatives may require much more time for the first phases of the action research cycle and this can prevent the completion of the specifying learning phase before the evaluation date. 


\section{CONCLUSION}

This paper has introduced the concept of an ability based framework, utilizing different input- and output modalities in conjunction with a mapping module, which optimally chooses the input- and output mechanisms based on a large number of trainee profile information (including learning style, abilities, perceptual and sensory preferences and literacy level).

The paper describes the action research methodology, which is used in this research project, and maps the various phases to concrete actions as currently being executed in the research project.

The action research approach has proven to be an effective methodology in guiding a complex research initiative, as described in this paper. When action research is used for a complex research initiative, care has to be taken to define the various phases of a cycle according to the evaluation dates, where possible. This will ensure that the necessary research outputs are completed before the required evaluation date.

\section{REFERENCES}

[1] G. Susman and R. Evered, "An assessment of the scientific merits of action research," Administrative Science Quarterly, vol. 23, no. 4, p. $582,1978$.

[2] L. Thomas, "Bums on seats; or listening to voices: evaluating widening participation initiatives using participatory action research."

[3] R. Baskervile and A. Wood-Harper, "A critical perspective on action research as a method for information systems research," Journal of Information Technology, vol. 11, no. 3, p. 235, 1996.

[4] R. Baskerville and J. Pries-Heje, "Grounded action research: a method for understanding it in practice," Accounting, Management and Information Technologies, vol. 9, no. 1, pp. 1-23, 1999.

[5] M. Blattner and E. Glinert, "Multimodal integration," Multimedia IEEE, vol. 3, no. 4, pp. 14-24, 1996.

[6] S. Keates and P. Robinson, "Gestures and multimodal input," Behaviour \& Information Technology, vol. 18, no. 1, p. 36, 1999.

[7] I. Rauschert, "Adaptive multimodal recognition of voluntary and involuntary gestures of people with motor disabilities," in Proceedings of the 6th international conference on Multimodal interfaces, Session: Doctoral spotlight, State College, U.S.A., 2004, p. 356.

[8] A. S. et al., "Multimodal input for computer access and augmentative communication," in Proceedings of the second annual ACM conference on Assistive technologies, Vancouver, British Columbia, Canada, 1996, pp. 80-85.

[9] S. Wall and S. Brewster, "Feeling what you hear: tactile feedback for navigation of audio graphs," in Proceedings of the SIGCHI conference on Human Factors in computing systems, Session: Disabilities, Montreal, Qu'ebec, Canada, 2006, pp. 1123-1132.

[10] - "Tac-tiles: Multimodal pie charts for visually impaired users," in Proceedings of the 4th Nordic conference on Human-computer interaction: changing roles, Oslo, Norway, 2006, pp. 9-18.
[11] S. Münch and R. Dillmann, "Haptic output in multimodal user interfaces," in Proceedings of the 2nd international conference on Intelligent user interfaces, Orlando, Florida, U.S.A., 1997, pp. 105-112.

[12] S. Oviatt, "Designing robust multimodal systems for universal access," in Proccedings of the 2001 EC/NSF workshop on Universal accessibility of ubiquitous computing: providing for the elderly table of contents, Alc'acer do Sal, Portugal, 2001, pp. 71-74.

[13] S. Kawai, H. Aida, and T.Saito, "Designing interface toolkit with dynamic selectable modality," in Proceedings of the second annual ACM conference on Assistive technologies, Vancouver, British Columbia, Canada, 1996, pp. 72-79.

[14] F. A. et al, "The aspice project: inclusive design for the motor disabled," in Proceedings of the working conference on Advanced visual interfaces, Venezia, Italy, 2006, pp. 360-363.

[15] R. Rochford, "Assessing learning styles to improve the quality of performance of community college students in developmental writing programs: a pilot study," Community College Journal of Research and Practice, vol. 27, pp. 665-677, 2003.

[16] C. VanEtten and G. VanEtten, "A working model for developing instructional materials for the learning disabled," Learning Disability Quarterly, vol. 1, no. 2, pp. 32-42, 1978.

[17] K. Sandberg, A. Gardelli, and J. Stubbs, "The use of information and communication technology (ict) in the rehabilitation of individuals with severe functional impairments in a municipal care service system," Work, vol. 24, no. 3, pp. 229-238, 2005.

[18] A. Bosseler and D. Massaro, "Development and evaluation of a computer-animated tutor for vocabulary and language learning in children with autism," Journal of autism and developmental disorders, vol. 33, no. 6, December 2003.

[19] L. E. et al., "Teach-m: a pilot study evaluating an instructional sequence for persons with impaired memory and executive functions," Brain Injury, vol. 19, no. 8, pp. 569-583, August 2005.

[20] G. Douglas, "Ict, education and visual impairment," British Journal of Educational Technology, vol. 32, no. 3, pp. 353-364, 2001.

[21] J. Egan, L. Worrall, and D. Oxenham, "An internet training intervention for people with traumatic brain injury: barriers and outcomes," Brain Injury, vol. 19, no. 8, pp. 555-568, August 2005.

[22] K. Kabassi and M. Virvou, "Personalised adult e-training on computer use based on multiple attribute decision making," Interacting with computers, vol. 16, no. 1, pp. 115-132, 2004.

[23] C. Doulgeraki, N. Partarakis, A. Mourouzis, and C. Stephanidis, "Adaptable web-based user interfaces: methodology and practice," $e$-Minds: International Journal on Human-Computer Interaction, vol. 1, no. 5, pp. 79-110, 2009.

[24] G. Hearn and M. Foth, Topical issues in communications and media research. Nova Science, 2005, ch. Action Research in the Design of New Media and ICT Systems.

[25] M. Chetty, W. Tucker, and E. Blake, "Using voice over ip to bridge the digital divide - a critical action research approach," in Proceedings of South African telecommunications and Networking Application Conference (SATNAC), George, South Africa, 2003.

[26] R. Rapoport, "Three dilemmas in action research," Human Relations, vol. 23, no. 6, pp. 499-513, 1970. 